\title{
The efficacy and toxicity of individualized intensity-modulated radiotherapy based on the tumor extension patterns of nasopharyngeal carcinoma
}

\author{
Li Lin ${ }^{1, *}$, Ji-Jin Yao ${ }^{1, *}$, Guan-Qun Zhou ${ }^{1}$, Rui Guo ${ }^{1}$, Fan Zhang ${ }^{1}$, Yuan Zhang ${ }^{1}$, Lin Xu ${ }^{1}$, \\ Lu-Lu Zhang ${ }^{1}$, Ai-Hua Lin ${ }^{2}$, Jun Ma ${ }^{1}$, Ying Sun ${ }^{1}$ \\ ${ }^{1}$ Department of Radiation Oncology, Sun Yat-Sen University Cancer Center, State Key Laboratory of Oncology in South China, \\ Collaborative Innovation Center for Cancer Medicine, Guangzhou 510060, People's Republic of China \\ ${ }^{2}$ Department of Medical Statistics and Epidemiology, School of Public Health, Sun Yat-sen University, Guangzhou 510080, \\ People's Republic of China \\ *These authors have contributed equally to this work
}

Correspondence to: Ying Sun, e-mail: sunying@sysucc.org.cn

Keywords: nasopharyngeal carcinoma, intensity modulated radiotherapy, individualized clinical target volume, clinical outcome, toxicities

Received: October 10, 2015

Accepted: February 18, 2016

Published: March 09, 2016

\section{ABSTRACT}

Background: To evaluate the efficacy and toxicity of intensity-modulated radiotherapy (IMRT) using individualized clinical target volumes (CTVs) based on the loco-regional extension patterns of nasopharyngeal carcinoma (NPC).

Methods: From December 2009 to February 2012, 220 patients with histologically-proven, non-disseminated NPC were prospectively treated with IMRT according to an individualized delineation protocol. CTV1 encompassed the gross tumor volume, entire nasopharyngeal mucosa and structures within the pharyngobasilar fascia with a margin. CTV2 encompassed bilateral high risk anatomic sites and downstream anatomic sites adjacent to primary tumor, bilateral retropharyngeal regions, levels II, III and Va, and prophylactic irradiation was gave to one or two levels beyond clinical lymph nodes involvement. Clinical outcomes and toxicities were evaluated.

Results: Median follow-up was 50.8(range, 1.3-68.0) months, four-year local relapse-free, regional relapse-free, distant metastasis-free, disease-free and overall survival rates were $94.7 \%, 97.0 \%, 91.7 \%, 87.2 \%$ and $91.9 \%$, respectively. Acute severe ( $\geq$ grade 3 ) mucositis, dermatitis and xerostomia were observed in $27.6 \%$, $3.6 \%$ and zero patients, respectively. At 1 year, xerostomia was mild, with frequencies of Grade 0, 1, 2 and 3 xerostomia of $27.9 \%, 63.3 \%, 8.3 \%$ and $0.5 \%$, respectively.

Conclusions: IMRT using individualized CTVs provided high rates of local and regional control and a favorable toxicity profile in NPC. Individualized CTV delineation strategy is a promising one that may effectively avoid unnecessary or missed irradiation, and deserve optimization to define more precise individualized CTVs.

\section{INTRODUCTION}

Nasopharyngeal carcinoma is most endemic in South-Eastern Asians, with an age-standardized incidence in male of 20-50/100000 in southern china [1]. Radiation therapy is the mainstay treatment modality for non-metastatic disease. For decades, NPC radiation therapy utilizes two-dimensional conventional treatment (2D-CRT). Disease control has been acceptable [2-4]; however, high-dose irradiation has been associated with high probability of toxicities. Currently, intensity modulated radiotherapy (IMRT) is generally accepted as a more advanced radiation technique to improve the therapeutic ratio and encouraging outcomes have been achieved over the past decade [5-8].

The delineation of ideal target volumes is absolutely one of the most critical procedures in IMRT. However, 
optimal clinical target volume (CTV) for NPC is far away from determined. In the current practise of most institutions, anatomic sites surrounding the nasopharynx were empirically delineated bilaterally as CTV, regardless of the tumor extension patterns. Even though the CTV vary significantly among institutions, the local and regional control were comparably satisfactory, and outside-field loco-regional failures were fairly rare [9-14]. We speculate that current CTVs are large enough to encompass microscopic spread; but unnecessary irradiation and side effects may be existed [15-17]. With the improved survival in modern diagnostic and therapeutic modality of NPC, adequate quality of life after treatment is essential for patients and their family. Consequently, optimal target definition in 3D planning of IMRT is imperative to transform the technical advantages of IMRT to improved efficacy and toxicity profile.

In addition, current CTV delineation largely originated from experience in 2D-CRT rather than the loco-regional extension patterns of this malignancy. Our previous studies have investigated the patterns of local extension and cervical lymph node (LN) metastasis $[18,19]$, which was fundamental in understanding the biological characteristics of NPC and could help to define individualized CTV avoiding unnecessary or missed irradiation.

Here, we generated an individualized CTV delineation protocol based on the loco-regional extension patterns of NPC. The aim of this work was to report the efficacy and toxicity of a cohort of patients perceptively treated with individualized IMRT and to explore the feasibility of individualized CTV delineation in NPC.

\section{RESULTS}

\section{Dose-volume analysis for targets and organs at risk}

Table 1 shows the dose-volume statistics for targets and organs at risk (OARs). The mean dose to planning target volume of nasopharynx (PTVnx) was 72.62 (range, 70.77-74.52) Gray (Gy). On average, target volumes had excellent dosimetric distributions. The prescribed dose encompassed $98.84 \%$ of PTVnx, $99.43 \%$ of planning target volume 1 (PTV1) and $98.54 \%$ of planning target volume 2 (PTV2); only $5.33 \%$ of PTVnx received $\geq 110 \%$ of the prescribed dose. The dose received by $1 \%$ of the volume of the planning organ at risk volume (PRV) of the brain stem and spinal cord was 58.64 Gy and 39.87 Gy, respectively. The doses delivered were within the tolerance limits of most OARs, except bilateral parotid glands.

\section{Treatment outcomes}

All patients completed the scheduled radiotherapy. The median follow-up was 50.8 (range, 1.3-68.0) months,
$58.7 \%$ of patients were followed up for more than 4 years. Of the patients, 11 developed local relapse; 6, regional relapse; 18, distant metastasis; and 19 died. Four-year estimated local relapse free survival (LRFS), regional relapse free survival (RRFS), distant metastasis free survival (DMFS), disease free survival (DFS) and overall survival (OS) rates were 94.7\% (95\% Confidence Interval, CI: $91.8 \%-97.6 \%$ ), 97.0\% (95\%CI: 94.7\%-99.4\%), 91.7\% (95\%CI: 87.9\%-95.4\%), 87.2\% (95\%CI: 82.7\%-91.7\%) and $91.9 \%(95 \% \mathrm{CI}: 88.2 \%-9.6 \%)$, respectively (Figure 1). Thirteen deaths were due to distant metastasis; 5, progression of loco-regional disease after recurrence; and 1 , an unrelated accident.

Median time to loco-regional recurrence was 23.7 (range, 4.5-34.3) months. Ten of 11 cases with local recurrence occurred within the $95 \%$ isodose lines of PTVnx and were considered in-field failures; the other case recurred at the initial primary site with extension out of PTVnx and was considered marginal failure. No recurrence was observed outside the margin of CTV2. MRI images obtained before treatment and at the time of recurrence of primary tumor of 11 patients with local recurrence are show in Supplementary Figure S1A-S1E. Most local relapses $(9 / 11)$ occurred in patients with locally advanced disease.

Of the 6 regional recurrences, 5 occurred in level $\mathrm{IIb}$ where metastatic nodes were formerly present; the other, in level Ia and Ib that haven't been irradiated before at 23.3 months, and was considered out-of-field failure. Five of the 6 regional recurrences occurred with local recurrence. Four-year RRFS rates of N0 and LN-positive patients were $100 \%$ and $96.5 \%$, respectively.

\section{Prognostic factors}

The value of potential prognostic factors including age, gender, $\mathrm{T}$ classification, $\mathrm{N}$ classification, WHO histological grade and chemotherapy were evaluated using Cox proportional hazards models with backward elimination. The $\mathrm{T}$ classification was found to be an independent predictor for OS $(P=0.008 ; \mathrm{HR}=2.29 ; 95 \%$ $\mathrm{CI}=1.24-4.214)$, whereas all the other prognostic factors were found to be insignificant (Table 2).

\section{Acute and late radiation toxicities}

Acute toxicities were assessed during treatment in 220 patients and late toxicities in 218 patients with $\geq 6$ months follow-up (Table 3). Acute toxicities of radiotherapy \pm chemotherapy were well tolerated. Acute severe ( $\geq$ Grade 3 ) mucositis, dermatitis and xerostomia were observed in $27.6 \%, 3.6 \%$ and zero patients, respectively. Xerostomia at 1 year was mild, with frequencies of Grade 0, 1, 2 and 3 xerostomia of $27.9 \%$, $63.3 \%, 8.3 \%$ and $0.5 \%$, respectively. Fourteen patients (6.4\%) developed temporal lobe injury diagnosed by MRI after IMRT. 
Table 1: Dose-volume data for the targets and critical organs at risk (OARs)

\begin{tabular}{|c|c|c|c|c|c|c|}
\hline & Structure & $\begin{array}{l}\text { Parameters } \\
\text { (unit) }\end{array}$ & Constraints & $\begin{array}{c}\text { Result } \\
\text { Mean (range) }\end{array}$ & $\begin{array}{c}\text { Dmean (Gy) } \\
\text { Mean (range) }\end{array}$ & $\begin{array}{l}\text { Volume (cc) }{ }^{\#} \\
\text { Mean (range) }\end{array}$ \\
\hline \multirow[t]{5}{*}{ Targets } & PTVnx & V95\% $(\%)^{*}$ & $\geq 99$ & $99.85(96.9-100.00)$ & $72.62(70.77-74.52)$ & $44.71(0.89-246.60)$ \\
\hline & & V110\% $(\%)^{*}$ & $\leq 20$ & $5.33(0-44.6)$ & & \\
\hline & PTV1 & V95\% (\%) & $\geq 99 \%$ & $99.92(99.00-100.00)$ & $68.76(65.45-71.33)$ & $124.07(21.21-448.86)$ \\
\hline & & $\mathrm{V} 100 \%(\%)$ & $\geq 95 \%$ & $99.43(96.90-100.00)$ & & \\
\hline & & $\mathrm{V} 100 \%(\%)$ & $\geq 95 \%$ & $98.54(91.91-99.94)$ & & \\
\hline \multirow[t]{10}{*}{ OARs } & Brainstem & $\operatorname{Dmax}(\mathrm{Gy}) \dagger$ & $\leq 54$ & $54.10(40.95-69.40)$ & & \\
\hline & Brainstem PRV & $\mathrm{D} 1 \%(\mathrm{~Gy}) \dagger \dagger$ & $\leq 60$ & $58.64(47.94-74.40)$ & & \\
\hline & Spinal cord & Dmax (Gy) & $\leq 45$ & $37.92(33.38-48.95)$ & & \\
\hline & Spinal cord PRV & $\mathrm{D} 1 \%(\mathrm{~Gy})$ & $\leq 50$ & $39.87(35.00-51.18)$ & & \\
\hline & Left temporal lobe & $\operatorname{Dmax}(\mathrm{Gy})$ & $\leq 60$ & $60.15(41.97-74.95)$ & & \\
\hline & $\begin{array}{l}\text { Right temporal } \\
\text { lobe }\end{array}$ & Dmax (Gy) & $\leq 60$ & $59.93(42.10-75.47)$ & & \\
\hline & Left parotid & Dmean $(\mathrm{Gy}) \ddagger$ & $\leq 26$ & $39.18(28.94-63.20)$ & & \\
\hline & & V30Gy $(\%)$ व & $\leq 50$ & $60.03(24.60-100.00)$ & & \\
\hline & Right parotid & Dmean (Gy) & $\leq 26$ & $38.90(30.14-61.22)$ & & \\
\hline & & V30Gy (\%) & $\leq 50$ & $58.54(29.20-100)$ & & \\
\hline
\end{tabular}

*Percentage volume receiving $\geq 95 \%, 100 \%$ or $110 \%$ of the prescribed dose;

${ }^{\dagger}$ Maximum dose;

${ }^{\dagger}$ Dose received by $1 \%$ of the volume concerned;

\$ean dose;

"Percentage volume receiving $\geq 30 \mathrm{~Gy}$;

"Volumes of GTVnx, CTV1 and CTV2.

\section{DISCUSSION}

This report summarizes the efficacy and toxicity of IMRT using an individualized CTV delineation protocol based on the patterns of tumor extension and biological characteristics of NPC. Up to now, the optimal CTV delineation scheme remains to be identified, and our work was the first attempt to explore the feasibility of individualized CTV delineation during IMRT planning. Using individualized CTV, we achieved a 4-year LRFS, RRFS, DMFS, DFS and OS of 94.7\%, 97.0\%, 91.7\%, $87.2 \%$ and $91.9 \%$, respectively, comparable to the historical data [9-14]. Excellent outcomes supported the feasibility of individualized CTV delineation.

\section{Feasibility of individualized CTV delineation for local disease}

It is difficult to gain pathological confirmation of tumor extension in NPC as it is unresectable. Thus, imaging concerned tumor extension patterns are critical for CTV delineation. According to the cumulative incidences of tumor invasion, Liang et al. and $\mathrm{Li}$ et al. classified the anatomic sites surrounding the nasopharynx into three risk grades $[18,20]$. The high risk anatomic sites are all adjacent to the nasopharynx and have a cumulative involvement probability $>30 \%$. Thus, it is reasonable to speculate that these high risk anatomic sites are indeed at high risk of tumor invasion; and should be included in CTV2 in all patients. As the risk of tumor invasion gradually reduces with the distance from the nasopharynx, it seems reasonable to subdivide the CTV into CTV1 and CTV2 with different dose levels to different risk regions. In many treatment centers, only one CTV for the primary tumor is delineated, and the region is similar to that of our CTV2; however, the dose prescribed is the same as that to our CTV1 $[9-11,14]$. The comparable local control rates indicate it is safe to narrow the area irradiated at $60 \mathrm{~Gy}$ and prescribe a lower dose to CTV2. 
Medium and low risk anatomic sites are separated from the nasopharynx by other anatomic sites and are rarely invaded without involvement of adjacent high or medium risk anatomic sites $[18,20]$. In most studies, medium or low risk anatomic sites including the bilateral foramen ovale, pterygopalatine fossa, sphenoid sinus, and posterior part of the maxillary sinus are regularly included in CTV. This strategy seems unreasonable, especially in T1 or T2 disease, due to the risk of unnecessary irradiation. In current practice, prophylactic radiation is prescribed along the tumor infiltration routes to include downstream anatomic sites adjacent to the tumor, in order to achieve individualized treatment. Accordingly, the aforementioned medium or low risk anatomic sites would be omitted in patients with tumors restricted to the pharyngobasilar fascia. In current series, ten patients experience local recurrence within the delineated GTV, and 1 patient have marginal local recurrence with a component of recurrent foci in the GTV. Isolated recurrence at the edge of the delineated CTV2 was not seen. Individualized CTV for primary disease described above seems to be feasible for radiation therapy for NPC using IMRT.

\section{Feasibility of individualized CTV delineation for regional disease}

Due to the high probability of cervical lymph node metastasis [21], irradiation of the entire neck down to the supraclavicular fossae (SCF) is commonly-accepted practice, even in N0 disease [22]. Recent studies reported that metastasis to the cervical LNs follows orderly pattern, with rarely skipping LN levels [19, 20,23]. Consequently, it is reasonable to question the necessity of elective irradiation to the lower neck (such as levels IV and $\mathrm{Vb}$ ) and SCF nodes in patients with N0 disease. Tang et al. retrospectively compared 138 patients had N0 disease with (101 patients) or without (37 patients) lower neck irradiation [19]. None of the patients in either group experienced regional failure, and the risks of distant metastasis did not differ statistically. Furthermore, in a study reported by Gao et al., 410 NPC patients with N0 disease were treated with conventional radiotherapy, but only lymph nodes in the upper neck nodes were electively irradiated [24]. At 5-year follow-up, only one case (0.2\%) of regional recurrence in the lower neck was observed. In current series, 30 patients had N0 disease and were irradiated only to the caudal border of the cricoid bone, no patient experienced regional failure. These results suggested that in patients with N0 disease, spearing the lower neck may be acceptable with a minimal risk of regional recurrence. However, further investigations are needed to confirm the findings before the limited neck field becomes the standard of CTV delineation in IMRT for NPC patients with N0 disease.

Currently, most studies advocate treatment of whole neck including level IV, Vb, and SCF in patients with LN-positive patients, regardless of where the nodes were emerged [3, 12-14], Owing to the low risk of "skip
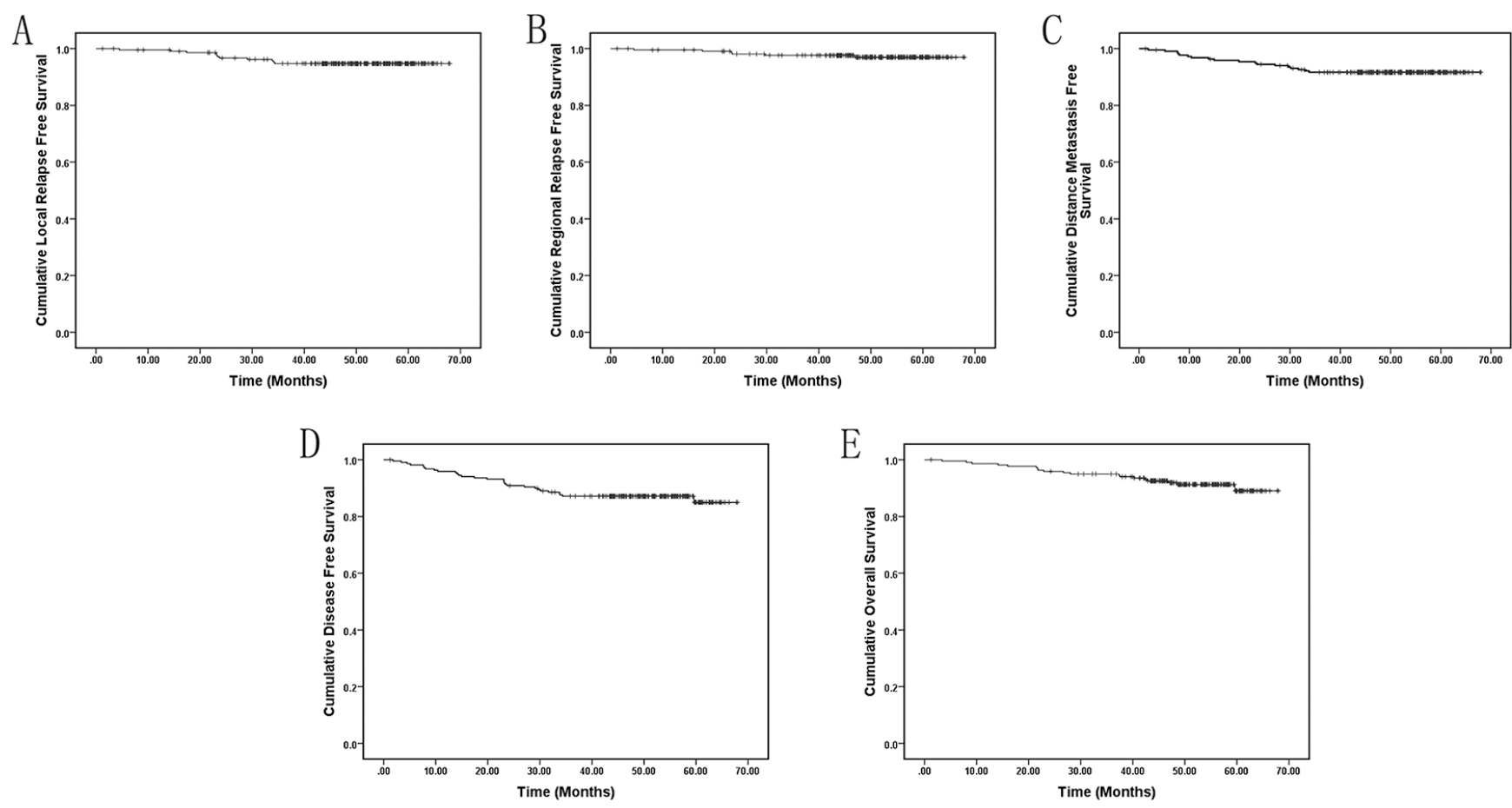

Figure 1: Kaplan-Meier local relapse free (A), regional relapse-free (B), distant metastasis-free (C), disease-free (D), and overall (E) survival curves. 
Table 2: Multivariate analyses of prognostic factors

\begin{tabular}{|c|c|c|c|c|c|}
\hline \multirow{2}{*}{ Variable } & \multicolumn{5}{|c|}{ HR (95\% CI), $P$-value } \\
\hline & LRFS & RRFS & DMFS & DFS & OS \\
\hline \multirow[t]{2}{*}{ Gender, male $v s$. female } & $1.28(0.22-7.56)$ & $1.51(0.43-5.34)$ & $0.61(0.18-2.12)$ & $0.93(0.39-2.20)$ & $0.56(0.16-1.94)$ \\
\hline & $P=.78$ & $P=.53$ & $P=.44$ & $P=.86$ & $P=.36$ \\
\hline \multirow{2}{*}{ Age, $\geq 50 v s .<50$ years } & $1.11(0.20-6.28)$ & $0.53(0.11-2.43)$ & $1.42(0.55-3.68)$ & $1.35(0.63-2.87)$ & $1.77(0.71-4.42)$ \\
\hline & $P=.90$ & $P=.41$ & $P=.47$ & $P=.44$ & $P=.22$ \\
\hline \multirow{2}{*}{$\begin{array}{l}\text { T classification, } \\
\text { T4 vs. T3 vs. T2 vs. T1 }\end{array}$} & $0.88(0.39-1.99)$ & $1.06(0.57-1.96)$ & $1.44(0.86-2.43)$ & $1.32(0.89-1.97)$ & $2.29(1.24-4.21)$ \\
\hline & $P=.75$ & $P=.85$ & $P=.17$ & $P=.17$ & $P=.008$ \\
\hline \multirow{2}{*}{$\begin{array}{l}\mathrm{N} \text { classification, } \\
\mathrm{N} 3 v_{s} . \mathrm{N} 2 v_{s} . \mathrm{N} 1 \text { vs. N0 }\end{array}$} & $1.60(0.84-3.04)$ & $1.46(0.87-2.44)$ & $1.24(0.80-1.92)$ & $1.37(0.99-1.90)$ & $1.37(0.92-2.03)$ \\
\hline & $P=.15$ & $P=.15$ & $P=.34$ & $P=.056$ & $P=.12$ \\
\hline \multirow{2}{*}{$\begin{array}{l}\text { WHO histological grade, } \\
\text { Type III } v \text { s. Type I and II }\end{array}$} & - & - & $1.53(0.34-6.89)$ & $0.83(0.20-3.54)$ & $0.48(0.06-3.78)$ \\
\hline & - & - & $P=.58$ & $P=.81$ & $P=.49$ \\
\hline \multirow{2}{*}{$\begin{array}{l}\text { Chemotherapy, } \\
\text { with vs. without }\end{array}$} & $0.50(0.04-5.67)$ & $0.90(0.10-8.50)$ & $1.98(0.24-16.22)$ & $0.86(0.24-3.16)$ & $0.56(0.11-2.63)$ \\
\hline & $P=.58$ & $P=.92$ & $P=.52$ & $P=.83$ & $P=.46$ \\
\hline
\end{tabular}

Abbreviations: HR, hazards ratio; 95\% CI, 95\% confidence interval; RT, radiotherapy; CRT, chemoradiotherapy; LRFS, local relapse free survival; RRFS, regional relapse free survival; DMFS, distance metastasis free survival; DFS, disease free survival; OS, overall survival.

\section{Table 3: Frequencies of the most common acute and late radiation toxicities by type and grade}

\begin{tabular}{|c|c|c|c|c|c|}
\hline Treatment toxicities & Total & Grade 1 & Grade 2 & Grade 3 & Grade 4 \\
\hline \multicolumn{6}{|l|}{ Acute toxicities, $n$ (\%) } \\
\hline Mucositis & $219(99.0)$ & $53(24.0)$ & $105(47.4)$ & $61(27.6)$ & 0 \\
\hline Dermatitis & $215(97.0)$ & $146(65.8)$ & $61(27.6)$ & $8(3.6)$ & 0 \\
\hline Xerostomia & $205(92.8)$ & $143(64.8)$ & $62(28.0)$ & 0 & 0 \\
\hline \multicolumn{6}{|l|}{ Late toxicities, $n(\%)$} \\
\hline Xerostomia* & $157(72.1)$ & $138(63.3)$ & $18(8.3)$ & $1(0.5)$ & 0 \\
\hline Hearing loss & $71(32.6)$ & $67(30.7)$ & $3(1.4)$ & $1(0.5)$ & 0 \\
\hline Neck fibrosis & $67(30.7)$ & $67(30.7)$ & 0 & 0 & 0 \\
\hline Radiation encephalopathy & $14(6.4)$ & $13(5.9)$ & $1(0.5)$ & 0 & 0 \\
\hline
\end{tabular}

*At 12 months after radiotherapy, 217 patients were evaluable.

metastasis" in NPC, we prophylactically irradiated one or two levels beyond the clinical extent of LN involvement. The 3-year RRFS rate for the LN-positive patients was $97.2 \%$, and none of the regional recurrences occurred in the spared region, although recurrence at level Ia and Ib were observed in one patient. As local recurrence coexisted, this case cannot exactly be classified as regional recurrence as previous radiotherapy may have modified lymphatic drainage. Most recently, Chen et al. reviewed 154 patients with only RLN metastasis, of whom 54 received partial neck irradiation to levels II, III and Va and 100 received whole neck irradiation [25]. The 5-year RRFS and DFS rates for the partial neck irradiation and whole neck irradiation groups were $98.1 \%$ vs. $98.0 \%(P=.882)$ and $87.0 \%$ vs. $77.0 \%$ $(P=.117)$. These results indicate that prospective studies aiming at determining the individualized prophylactic neck irradiation area according to the location of lymph nodes is quite imperative.

Using individualized CTVs, we achieved relatively good target coverage and normal tissue sparing, except for bilateral parotid glands. Although doses irradiated to both parotid glands exceeded the dose limit suggest by the RTOG [26], sever acute and late xerostomia was rare. The explanation may be that we contoured and evaluated the whole parotid gland, including the part overlapped the CTV. Other toxicities of radiotherapy \pm chemotherapy were also acceptable, even with extensive use of chemotherapy $(86.4 \%)$. These may due to good normal tissue sparing and appropriate supportive care.

Despite the favorable local and regional control, there is an important issue need to be addressed. Most anatomic sites surrounding the nasopharynx have considerable volumes, and tumor invasion of these anatomic sites can vary from microscopic foci to gross infiltration. It remains unclear if it is necessary to prophylactically irradiate the adjacent downstream sites when a small proportion of an anatomic site is invaded. To figure out this issue, we now launched a program, attempting to define the invasion risk for each pixel of the anatomic sites surrounding the nasopharynx, thus more precise individualized CTVs could be defined. 
Table 4: Clinicopathological characteristics of the entire series of 220 patients

\begin{tabular}{|c|c|}
\hline Characteristics & $n(\%)$ \\
\hline \multicolumn{2}{|l|}{ Patient factors } \\
\hline \multicolumn{2}{|l|}{ Age (years) } \\
\hline$\geq 50$ & $68(30.9)$ \\
\hline$<50$ & $152(69.1)$ \\
\hline \multicolumn{2}{|l|}{ Gender } \\
\hline Male & $167(75.9)$ \\
\hline Female & $53(24.1)$ \\
\hline \multicolumn{2}{|l|}{ Tumor factors } \\
\hline \multicolumn{2}{|l|}{ Histology } \\
\hline WHO type I & $2(0.9)$ \\
\hline WHO type II & $15(6.8)$ \\
\hline WHO type III & $203(92.3)$ \\
\hline \multicolumn{2}{|l|}{ Clinical stage } \\
\hline $\mathrm{I}$ & $10(4.5)$ \\
\hline II & $46(20.9)$ \\
\hline III & $106(48.2)$ \\
\hline IV & $58(26.4)$ \\
\hline \multicolumn{2}{|l|}{ T category } \\
\hline $\mathrm{T} 1$ & $39(17.7)$ \\
\hline $\mathrm{T} 2$ & $33(15.0)$ \\
\hline $\mathrm{T} 3$ & $103(46.8)$ \\
\hline $\mathrm{T} 4$ & $45(20.5)$ \\
\hline \multicolumn{2}{|l|}{ N category } \\
\hline N0 & $30(13.6)$ \\
\hline N1 & $132(60.0)$ \\
\hline $\mathrm{N} 2$ & $40(18.2)$ \\
\hline N3 & $18(8.2)$ \\
\hline \multicolumn{2}{|l|}{ Treatment factors } \\
\hline \multicolumn{2}{|l|}{ chemotherapy } \\
\hline Yes & $190(86.4)$ \\
\hline No & $30(13.6)$ \\
\hline
\end{tabular}

Abbreviations: $\mathrm{WHO}=$ World Health Organization;

IMRT = Intensity-modulated radiotherapy.

To summarize, our individualized CTV delineation protocol resulted in favorable clinical outcomes and is a promising strategy that may effectively avoid unnecessary or missed irradiation. Further optimization is warranted to provide more precise individualized CTVs and maximize the tumor killing and normal tissue protecting effect.

\section{MATERIALS AND METHODS}

\section{Patients}

From December 2009 to February 2012, 220 patients with newly histologically-proven, non-disseminated NPC were prospectively treated with IMRT according to individualized CTV delineation protocol. Eligible patients were aged $18-70$ years with histologically proven stage I to stage IVB nasopharyngeal carcinoma (according to the 7th edition of International Union Against Cancer (UICC)/American Joint Committee on Cancer (AJCC) staging system). All patients had Karnofsky scores of at least 70, and adequate bone marrow, liver, and renal function. Our exclusion criteria included previous chemotherapy, radiotherapy, or definitive surgery of the primary tumor or lymph node. We also excluded patients with previous malignancy, with present other active cancer, who were pregnant or lactating, who had unstable cardiac disease needing treatment, or who had hearing loss due to sensorineural deafness. The study was approved by the institutional review board (IRB) and all patients provided written informed consent. Clinicopathological characteristics are summarized in Table 4. 
All patients underwent pre-treatment evaluations including complete history, physical and endoscopic examination, hematology and biochemistry profiles, plasma Epstein-barr virus (EBV) DNA concentrations, magnetic resonance imaging (MRI) of nasopharynx and neck, electrocardiogram, chest radiography, abdominal ultrasonography, emission computed tomography (ECT) and dental evaluation. The 7th edition of International Union Against Cancer (UICC)/American Joint Committee on Cancer (AJCC) staging system was used for disease staging [27].

\section{IMRT techniques}

Patient setup and treatment planning CT scan were performed as formerly described [28]. Target volumes were delineated slice-by-slice on treatment planning CT scans according to an individualized delineation protocol, in accordance with International Commission on Radiation Units and Measurements reports 50 and 62 [29, 30].

Gross tumor volume (GTV) was determined from imaging, physical examination and endoscopy. The primary tumor along with enlarged retropharyngeal lymph nodes (RLNs) was defined as GTVnx, and the involved cervical LNs volume as GTVnd. Organs at risk (OARs) were contoured consistent with Sun's recommendation [31].

Clinical target volume (CTV) was delineated individually based on GTV, patterns of loco-regional extension and biological characteristics of NPC. The CTV for GTVnx included CTV1 for high risk regions and CTV2 for low risk regions of microscopic infiltration. CTV1 was defined as GTVnx plus a $5-10 \mathrm{~mm}$ margin, including the entire nasopharyngeal mucosa and structures within the pharyngobasilar fascia. For CTV2, firstly, bilateral anatomic sites at high risk, including parapharyngeal space, posterior part of nasal cavity, pterygoid process, prevertebral muscle, clivus, petrous apex, foramen lacerum and basis of sphenoid bone, were included in each patient (Figure 2). Then, downstream anatomic sites adjacent to involved sites along routes of tumor infiltration were prophylactically irradiated (Figure 3 ). Finally, the margin of both CTVs could be reduced to $2-3 \mathrm{~mm}$ at the sites of brain stem, spinal cord and temporal lobes and margins could be limited to exclude bone or air spaces not at risk of subclinical disease.

Prophylactic neck irradiation areas were also defined as CTV2; individualized irradiation was achieved by selective irradiation according to the patterns of LN metastasis and modes of anatomic lymphatic drainage $[19,20,22,32]$. Cervical LN levels were defined according to International Consensus Guidelines for LN levels [22, 32]. Firstly, the bilateral retropharyngeal regions, levels II, III, and Va were included in CTV2 in each patient. Then, prophylactic irradiation was given to one or two levels beyond the clinical extent of LN involvement in LN positive patients. That is, ipsilateral levels IV and $\mathrm{Vb}$ were included in CTV2 in LN-positive patients and ipsilateral SCF was irradiated in patients with lymphadenopathy beyond the caudal end of the hyoid bone. Finally, level Ib was only electively irradiated if: (1) level Ib LNs were involved, (2) there was extensive nodal disease on the ipsilateral IIa/IIb region or extracapsular extension of level IIa LNs, or (3) the hard palate or ipsilateral nasal cavity was grossly involved. The outermost boundary of the CTV2 should be at least $5 \mathrm{~mm}$ from GTVnd.

Planning target volumes (PTVnx, PTVnd, PTV1 and PTV2) were constructed automatically by expanding the corresponding target volumes (GTVnx, GTVnd, CTV1 and CTV2) in three dimensions by $3 \mathrm{~mm}$, allowing for setup variability. All the PTVs should not go outside of the skin surface. A $3 \mathrm{~mm}$ margin was added to the brainstem and spinal cord to form the planning organ at risk volume (PRV).

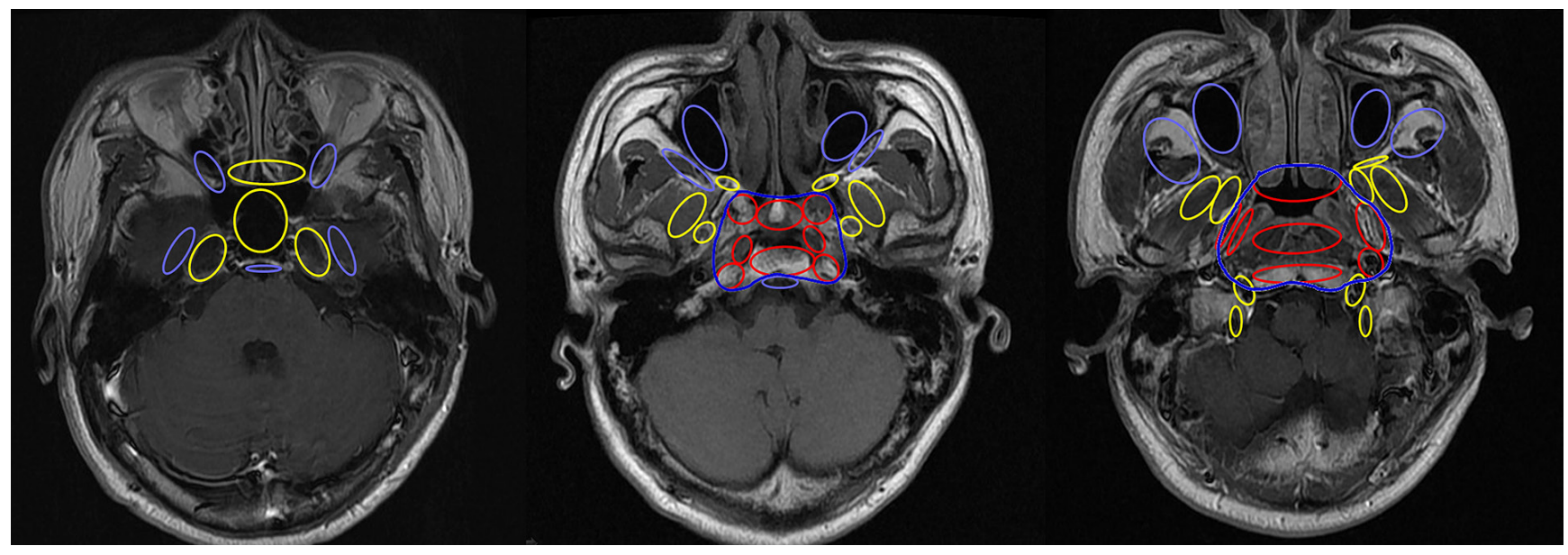

Figure 2: Relationship between the CTV2 and risk of tumor extension. Anatomic sites shown in red are at high risk of tumor invasion; yellow, medium risk; and light blue, low risk. The dark blue line represents the smallest CTV2 area for primary tumor. 


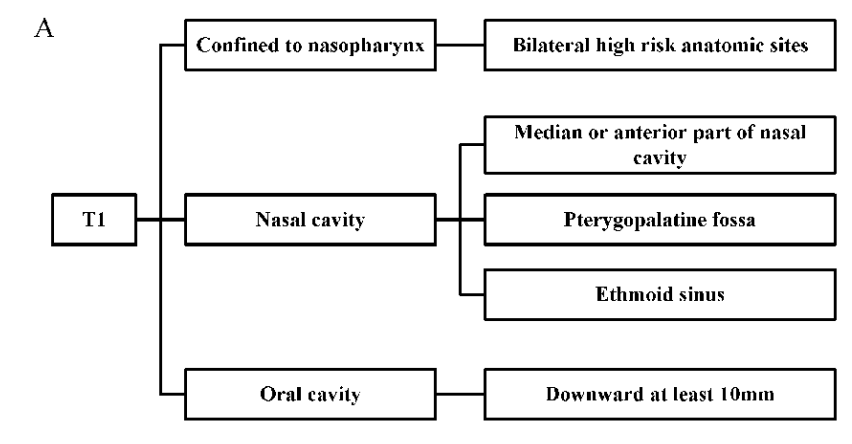

"

。

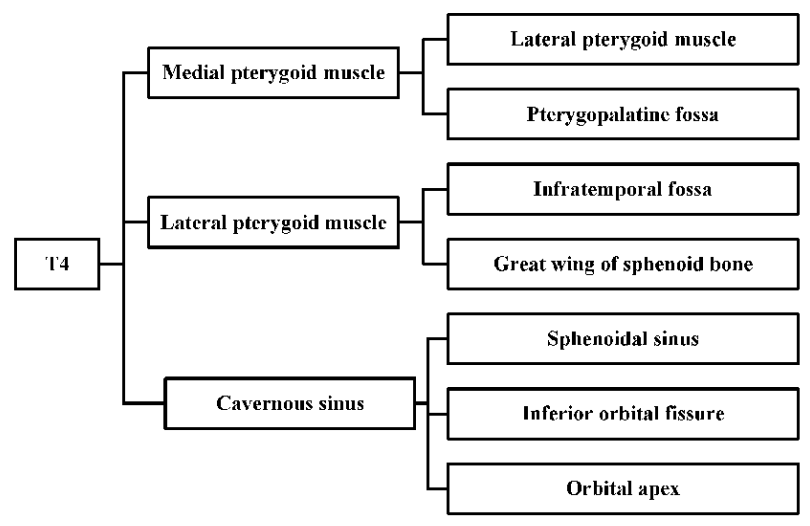

Tumor extension
Prophylactic irradiation region

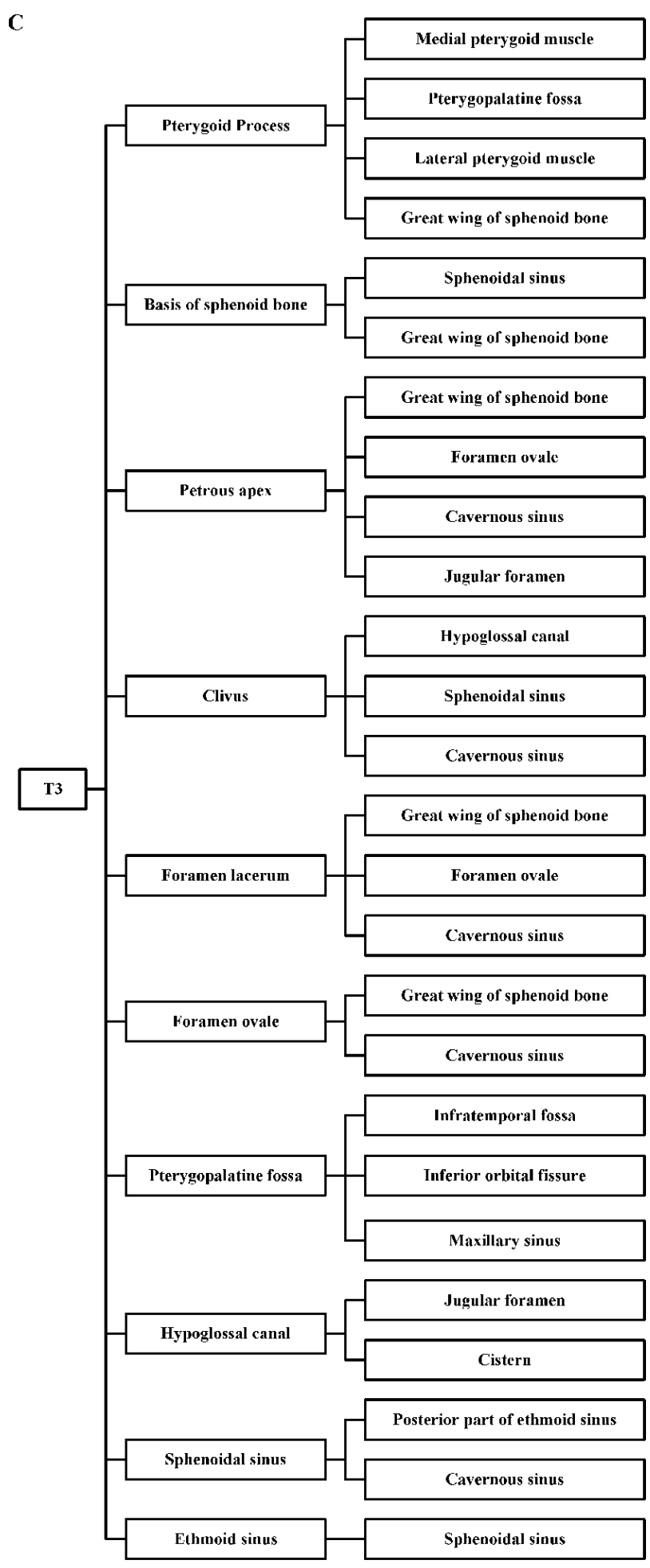

Tumor extension
Prophylactic irradiation region

Figure 3: Downstream anatomic sites to be prophylactically irradiated based on $T$ classification and routes of tumor extension. T1 (A), T2 (B), T3 (C), T4 (D). 
The prescribed doses were $68-70$ Gy at 2.12-2.27 Gy/fraction for PTVnx, 64-70 Gy at 2.00-2.13 Gy/ fraction for PTVnd, 60 Gy at 1.82-2.00 Gy/fraction for PTV1, and 54-56 Gy at 1.70-1.80 Gy/fraction for PTV2. The plan could be accepted if the target coverage met the criterion and dose receive by OARs should be as low as possible to met the restrictions (Table 2) [26]. All targets were treated simultaneously using the simultaneous integrated boost (SIB) technique. Treatment was delivered with a computer-controlled auto sequence multi-leaf collimator (MLC) on a linear accelerator. All treatments were delivered once daily, 5 days per week.

\section{Chemotherapy}

During the study, institutional guidelines recommended IMRT only for stage I and concurrent chemoradiotherapy \pm neoadjuvant/adjuvant chemotherapy for stage II to IVB. Overall, $30(13.6 \%)$ patients were treated with IMRT alone, and $190(86.4 \%)$ received chemotherapy. Of the 164 patients with stage III/IV disease, $155(94.5 \%)$ received chemotherapy Reasons for deviation included advanced age, organ dysfunction or allergic reactions. Neoadjuvant or adjuvant chemotherapy consisted of cisplatin with 5-fluorouracil and/or docetaxel every three weeks for two or three cycles. Concurrent chemotherapy consisted of cisplatin weekly or on days 1,22 and 43 of radiotherapy.

\section{Follow-up and statistical analysis}

All patients were evaluated at least per week during treatment and followed-up at least every three months in the first 2 years, every 6 months in the subsequent 3 years, and every year thereafter. Routine follow-up care included fiberoptic endoscopic examination, MRI of nasopharynx and neck, chest radiography, abdominal sonography, ECT and plasma EBV DNA concentrations.

Local relapse-free survival (LRFS), regional relapse-free survival (RRFS), distant metastasis-free survival (DMFS), disease-free survival (DFS) and overall survival (OS) rates were estimated by the Kaplan-Meier method. Durations were calculated from start of treatment. Radiotherapy-related toxicities were graded using the Acute and the Late Radiation Morbidity Scoring Criteria of the Radiation Therapy Oncology Group [33]. Multivariate analysis was performed using a Cox proportional hazards model. Analyses were performed using Statistical Package for the Social Sciences version 17.0 (SPSS; Chicago, IL, USA). Two-tailed $P$-values $\leq 0.05$ were considered significant.

\section{ACKNOWLEDGMENTS AND FUNDING}

This work was supported by grants from the Sun Yat-Sen University Clinical Research 5010 Program
(No. 2012011), the National Natural Science Foundation of China (No. 8172409), the National Science and Technology Pillar Program during the Twelfth Five-year Plan Period (2014BAI09B10), the Health and Medical Collaborative Innovation Project of Guangzhou City, China (201400000001).

\section{CONFLICTS OF INTEREST}

The authors indicate no actual or potential conflicts of interest exist.

\section{REFERENCES}

1. Jemal A, Bray F, Center MM, Ferlay J, Ward E, Forman D. Global cancer statistics. CA Cancer J Clin. 2011; 61:69-90.

2. Ma J, Mai HQ, Hong MH, Cui NJ, Lu TX, Lu LX, Mo HY, Min HQ. Is the 1997 AJCC staging system for nasopharyngeal carcinoma prognostically useful for Chinese patient populations? International journal of radiation oncology, biology, physics. 2001; 50:1181-1189.

3. Lee AW, Sze WM, Au JS, Leung SF, Leung TW, Chua DT, Zee BC, Law SC, Teo PM, Tung SY, Kwong DL, Lau WH. Treatment results for nasopharyngeal carcinoma in the modern era: the Hong Kong experience. International journal of radiation oncology, biology, physics. 2005; 61:1107-1116.

4. Leung TW, Tung SY, Sze WK, Wong FC, Yuen KK, Lui CM, Lo SH, Ng TY, O SK. Treatment results of 1070 patients with nasopharyngeal carcinoma: an analysis of survival and failure patterns. Head \& neck. 2005; 27:555-565.

5. Lai SZ, Li WF, Chen L, Luo W, Chen YY, Liu LZ, Sun Y, Lin AH, Liu MZ, Ma J. How does intensity-modulated radiotherapy versus conventional two-dimensional radiotherapy influence the treatment results in nasopharyngeal carcinoma patients? International journal of radiation oncology, biology, physics. 2011; 80:661-668.

6. Xiao WW, Huang SM, Han F, Wu SX, Lu LX, Lin CG, Deng XW, Lu TX, Cui NJ, Zhao C. Local control, survival, and late toxicities of locally advanced nasopharyngeal carcinoma treated by simultaneous modulated accelerated radiotherapy combined with cisplatin concurrent chemotherapy: long-term results of a phase 2 study. Cancer. 2011; 117:1874-1883.

7. Peng G, Wang T, Yang KY, Zhang S, Zhang T, Li Q, Han J, Wu G. A prospective, randomized study comparing outcomes and toxicities of intensity-modulated radiotherapy vs. conventional two-dimensional radiotherapy for the treatment of nasopharyngeal carcinoma. Radiotherapy and oncology. 2012; 104:286-293.

8. Sun X, Su S, Chen C, Han F, Zhao C, Xiao W, Deng X, Huang S, Lin C, Lu T. Long-term outcomes of intensity-modulated radiotherapy for 868 patients with nasopharyngeal carcinoma: an analysis of survival and treatment toxicities. Radiotherapy and oncology. 2014; 110:398-403. 
9. Lee N, Xia P, Quivey JM, Sultanem K, Poon I, Akazawa C, Akazawa P, Weinberg V, Fu KK. Intensity-modulated radiotherapy in the treatment of nasopharyngeal carcinoma: an update of the UCSF experience. International journal of radiation oncology, biology, physics. 2002; 53:12-22.

10. Kam MK, Teo PM, Chau RM, Cheung KY, Choi PH, Kwan WH, Leung SF, Zee B, Chan AT. Treatment of nasopharyngeal carcinoma with intensity-modulated radiotherapy: the Hong Kong experience. International journal of radiation oncology, biology, physics. 2004; 60:1440-1450.

11. Wolden SL, Chen WC, Pfister DG, Kraus DH, Berry SL, Zelefsky MJ. Intensity-modulated radiation therapy (IMRT) for nasopharynx cancer: update of the Memorial Sloan-Kettering experience. International journal of radiation oncology, biology, physics. 2006; 64:57-62.

12. Lee N, Harris J, Garden AS, Straube W, Glisson B, Xia P, Bosch W, Morrison WH, Quivey J, Thorstad W, Jones C, Ang KK. Intensity-modulated radiation therapy with or without chemotherapy for nasopharyngeal carcinoma: radiation therapy oncology group phase II trial 0225 . Journal of clinical oncology. 2009; 27:3684-3690.

13. Lin S, Pan J, Han L, Zhang X, Liao X, Lu JJ. Nasopharyngeal carcinoma treated with reduced-volume intensity-modulated radiation therapy: report on the 3-year outcome of a prospective series. International journal of radiation oncology, biology, physics. 2009; 75:1071-1078.

14. Tham IW, Hee SW, Yeo RM, Salleh PB, Lee J, Tan TW, Fong KW, Chua ET, Wee JT. Treatment of nasopharyngeal carcinoma using intensity-modulated radiotherapy-the national cancer centre singapore experience. International journal of radiation oncology, biology, physics. 2009; 75:1481-1486.

15. Kam MK, Leung SF, Zee B, Chau RM, Suen JJ, Mo F, Lai M, Ho R, Cheung KY, Yu BK, Chiu SK, Choi PH, Teo PM, et al. Prospective randomized study of intensity-modulated radiotherapy on salivary gland function in early-stage nasopharyngeal carcinoma patients. Journal of clinical oncology. 2007; 25:4873-4879.

16. Zhou GQ, Yu XL, Chen M, Guo R, Lei Y, Sun Y, Mao YP, Liu LZ, Li L, Lin AH, Ma J. Radiation-induced temporal lobe injury for nasopharyngeal carcinoma: a comparison of intensity-modulated radiotherapy and conventional two-dimensional radiotherapy. PloS one. 2013; 8:e67488.

17. Zeng L, Tian YM, Sun XM, Chen CY, Han F, Xiao WW, Deng XW, Lu TX. Late toxicities after intensity-modulated radiotherapy for nasopharyngeal carcinoma: patient and treatment-related risk factors. British journal of cancer. 2014; 110:49-54.

18. Liang SB, Sun Y, Liu LZ, Chen Y, Chen L, Mao YP, Tang LL, Tian L, Lin AH, Liu MZ, Li L, Ma J. Extension of local disease in nasopharyngeal carcinoma detected by magnetic resonance imaging: improvement of clinical target volume delineation. International journal of radiation oncology, biology, physics. 2009; 75:742-750.
19. Tang L, Mao Y, Liu L, Liang S, Chen Y, Sun Y, Liao X, Lin A, Liu M, Li L, Ma J. The volume to be irradiated during selective neck irradiation in nasopharyngeal carcinoma: analysis of the spread patterns in lymph nodes by magnetic resonance imaging. Cancer. 2009; 115:680-688.

20. Li WF, Sun Y, Chen M, Tang LL, Liu LZ, Mao YP, Chen L, Zhou GQ, Li L, Ma J. Locoregional extension patterns of nasopharyngeal carcinoma and suggestions for clinical target volume delineation. Chinese journal of cancer. 2012; 31:579-587.

21. Sham JS, Choy D, Wei WI. Nasopharyngeal carcinoma: orderly neck node spread. International journal of radiation oncology, biology, physics. 1990; 19:929-933.

22. Grégoire V, Levendag P, Ang KK, Bernier J, Braaksma M, Budach V, Chao C, Coche E, Cooper JS, Cosnard G, Eisbruch A, El-Sayed S, Emami B, et al. CT-based delineation of lymph node levels and related CTVs in the node-negative neck: DAHANCA, EORTC, GORTEC, NCIC,RTOG consensus guidelines. Radiotherapy and Oncology. 2003; 69:227-236.

23. Ng SH, Chang JT, Chan SC, Ko SF, Wang HM, Liao CT, Chang YC, Yen TC. Nodal metastases of nasopharyngeal carcinoma: patterns of disease on MRI and FDG PET. European journal of nuclear medicine and molecular imaging. 2004; 31:1073-1080.

24. Gao Y, Zhu G, Lu J, Ying H, Kong L, Wu Y, Hu C. Is elective irradiation to the lower neck necessary for N0 nasopharyngeal carcinoma? International journal of radiation oncology, biology, physics. 2010; 77: 1397-1402.

25. Chen M, Tang LL, Sun Y, Mao YP, Li WF, Guo R, Liu LZ, Li L, Lin AH, Ma J. Treatment outcomes and feasibility of partial neck irradiation for patients with nasopharyngeal carcinoma with only retropharyngeal lymph node metastasis after intensity-modulated radiotherapy. Head \& neck. 2014; 36:468-473.

26. Radiation Therapy Oncology Group Protocol 06-15. Available at: http://www.rtog.org/members/protocols/0615/ 0615.pdf. Accessed August 26, 2008.

27. SB E, DR B, CC C. (2010). AJCC Cancer Staging Manual, 7th Edition. In: Springer NY, ed.

28. Sun Y, Tang LL, Chen L, Li WF, Mao YP, Liu LZ, Lin AH, Li L, Ma J. Promising treatment outcomes of intensity-modulated radiation therapy for nasopharyngeal carcinoma patients with N0 disease according to the seventh edition of the AJCC staging system. BMC cancer. 2012; 12:68.

29. Definition of volumes. Journal of the ICRU. 2010; 10: 41-53.

30. Torre LA, Bray F, Siegel RL, Ferlay J, Lortet-Tieulent J, Jemal A. Global cancer statistics, 2012. CA Cancer J Clin. 2015; 65:87-108.

31. Sun Y, Yu XL, Luo W, Lee AW, Wee JT, Lee N, Zhou GQ, Tang LL, Tao CJ, Guo R, Mao YP, Zhang R, Guo Y, et al. Recommendation for a contouring method and atlas of organs at risk in nasopharyngeal carcinoma patients 
receiving intensity-modulated radiotherapy. Radiotherapy and oncology. 2014; 110:390-397.

32. Gregoire V, Eisbruch A, Hamoir M, Levendag P. Proposal for the delineation of the nodal CTV in the node-positive and the post-operative neck. Radiotherapy and oncology. 2006; 79:15-20.
33. Cox JD, Stetz J, Pajak TF. Toxicity criteria of the Radiation Therapy Oncology Group (RTOG) and the European Organization for Research and Treatment of Cancer (EORTC). International journal of radiation oncology, biology, physics. 1995; 31:1341-1346. 